\title{
Innovations en immunothérapie anticancéreuse : thérapie par lymphocytes $T$ porteurs de récepteurs antigéniques chimériques (cellules CAR-T)
}

\author{
Kevin Brown MD, Matthew D. Seftel MD MSP, Kevin A. Hay MD MSc
}

- Citation : CMAJ 2021 August 23;193:E1300-2. doi : 10.1503/cmaj.202907-f

Voir la version anglaise de l'article ici : www.cmaj.ca/lookup/doi/10.1503/cmaj.202907

$\mathbf{L}$ 'immunothérapie cellulaire est un traitement novateur qui fait appel aux propriétés anticancéreuses des cellules immunitaires du corps. L'une des techniques les plus prometteuses est la thérapie par lymphocytes T porteurs de récepteurs antigéniques chimériques (cellules CAR-T). Cette thérapie est aujourd'hui la norme en cas de leucémie lymphoblastique aiguë à cellules $B$ (LLA-B) récidivante ou réfractaire, le cancer le plus courant chez l'enfant, et de lymphome B de haut grade, le lymphome le plus fréquent au Canada ${ }^{1}$. La thérapie par cellules CAR-T fait actuellement l'objet d'essais cliniques pour le traitement d'autres cancers.

\section{Qu'est-ce que la thérapie par cellules CAR-T?}

Les lymphocytes $T$ jouent un rôle crucial dans la prévention du cancer. Les premières études cliniques utilisant des lymphocytes T non modifiés ont rapporté une efficacité modeste, mais la mise au point de techniques de génie génétique a permis aux chercheurs d'utiliser des vecteurs viraux non réplicatifs pour amener les lymphocytes $T$ à exprimer des « récepteurs antigéniques chimériques » (CAR). Cette intervention extérieure permet de cibler les antigènes cancéreux associés à l'activation des lymphocytes $T^{2}$. Une importante avancée a été réalisée lorsque la thérapie par cellules CAR-T dirigée contre le marqueur CD19 des lymphocytes $B$ a suscité une réponse clinique chez la plupart des patients ainsi qu'une réponse à long terme chez une petite proportion de patients présentant une LLA-B ou un lymphome $B$ de haut grade récidivants ou réfractaires. Cette découverte a amené la Food and Drug Administration des États-Unis et Santé Canada à approuver la thérapie par cellules CAR-T pour ces indications ${ }^{3}$.

\section{Comment cette thérapie est-elle administrée?}

Le processus général de modification des lymphocytes $T$ et d'administration de la thérapie est présenté à la figure 1 . Il existe divers produits thérapeutiques, qui ont chacun un vecteur viral et un processus de fabrication qui leur sont propres. Avant le début de la thérapie, les patients subissent une brève chimiothérapie

\section{Points clés}

- La thérapie par lymphocytes T porteurs de récepteurs antigéniques chimériques (cellules CAR-T) est un traitement cellulaire ciblé contre le cancer reposant sur la modification génique. Il est approuvé au Canada pour lutter contre la leucémie lymphoblastique aiguë à cellules B (LLA-B) et le lymphome $B$ de haut grade récidivants ou réfractaires.

- La plupart des patients qui présentent une leucémie lymphoblastique aiguë à cellules B (LLA-B) ou un lymphome B de haut grade récidivants ou réfractaires présentent de meilleurs taux de survie sans progression de la maladie et de survie globale que les témoins historiques, et une petite proportion de ces patients guérissent.

- La toxicité aiguë de la thérapie par cellules CAR-T peut prendre la forme d'un syndrome de relargage de cytokines ou d'un syndrome de neurotoxicité associé aux cellules immunitaires effectrices, et la toxicité à long terme, d'une cytopénie prolongée ou d'une hypoplasie lymphocytaire B.

- Des essais cliniques évaluant l'efficacité de la thérapie par cellules CAR-T contre d'autres cancer, y compris les tumeurs solides, sont en cours.

immunosuppressive, qui favorisera la prolifération des lymphocytes T modifiés après leur administration. Au Canada, la thérapie par cellules CAR-T est offerte dans les centres de transfusion sanguine et de greffe de moelle osseuse qui soignent les patients suivant un traitement cellulaire complexe.

\section{Qui peut recevoir cette thérapie?}

Au Canada, la thérapie par cellules CAR-T est approuvée pour les patients de 25 ans et moins dont la LLA-B est réfractaire au traitement de première intention, qui ont présenté une récidive après une greffe de cellules hématopoiétiques allogéniques ou qui ont connu au moins 2 récidives. Elle est également approuvée pour les adultes qui ont un lymphome $B$ de haut grade récidivant ou réfractaire et qui ont reçu au moins 2 traitements antérieurs. 


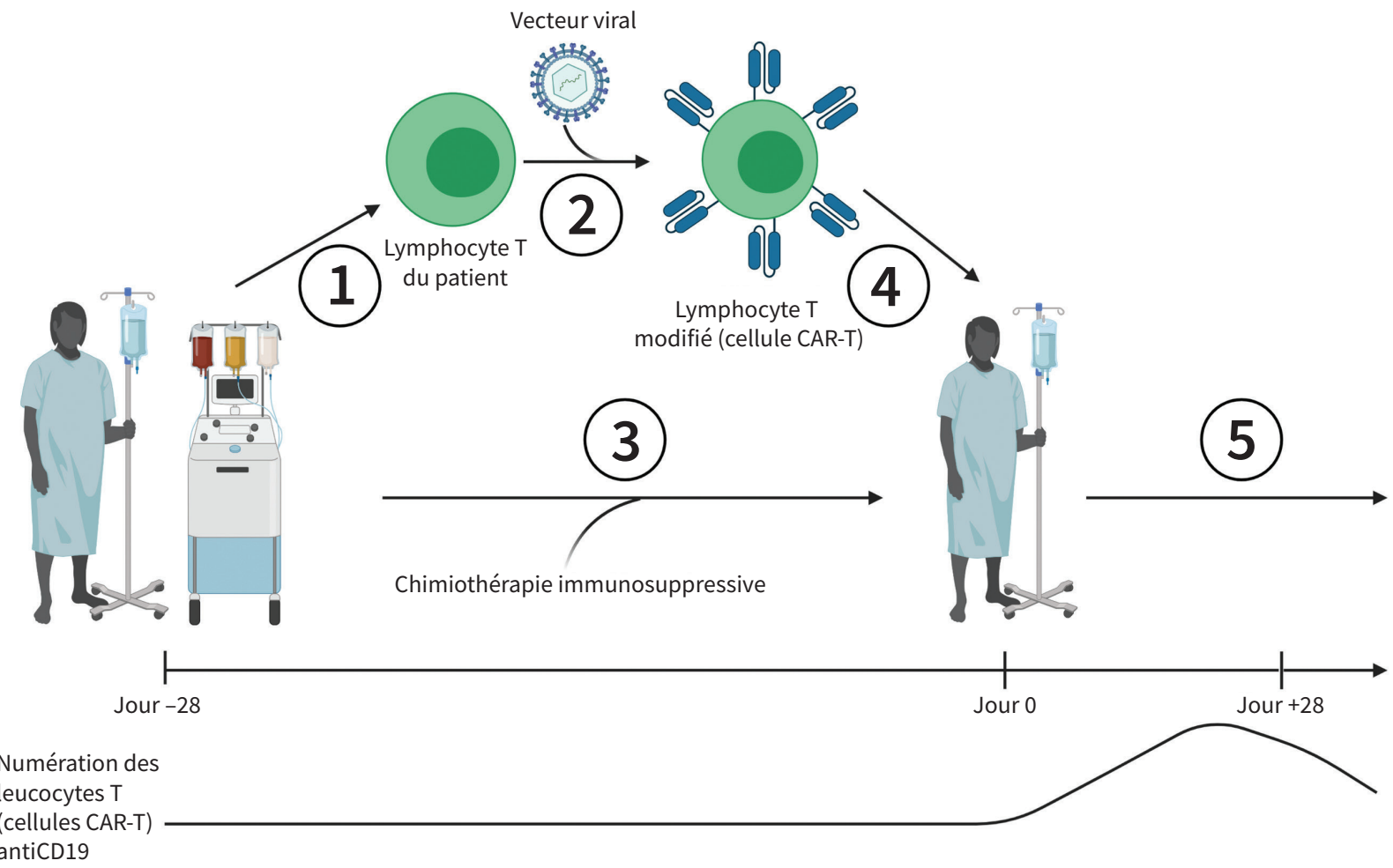

Figure 1: Processus général de modification des lymphocytes T et d'administration de la thérapie par lymphocytes T porteurs de récepteurs antigéniques chimériques (cellules CAR-T). 1) Les lymphocytes T du patient sont prélevés par aphérèse et transportés vers le centre, où ils seront modifiés. 2) Un vecteur viral est injecté dans les lymphocytes T du patient par transduction, qu'on laisse ensuite proliférer in vitro. 3) Pendant la production et la prolifération des lymphocytes $\mathrm{T}$, le patient reçoit une chimiothérapie immunosuppressive. 4) Les lymphocytes T modifiés sont transportés jusqu'au centre d'origine et administrés au patient. 5) Le patient fait l'objet d'un suivi de 28 jours visant à détecter l'apparition d'un syndrome de relargage de cytokines ou d'un syndrome de neurotoxicité associé aux cellules immunitaires effectrices. Un suivi à long terme est également réalisé pour surveiller l'apparition d'une hypoplasie lymphocytaire B, d'une hypogammaglobulinémie, d'une cytopénie ou d'une infection opportuniste. Une ligne du temps (non à l'échelle) indiquant les diverses étapes du processus et montrant la numération des lymphocytes T modifiés chez le patient est également présente.

\begin{tabular}{|c|c|c|c|}
\hline Produit & Maladie(s) traitée(s) & Antigène ciblé & Données sur la survie \\
\hline Tisagenlecleucel $\dagger^{4,5}$ & $\begin{array}{l}\text { LLA-B, lymphome B de haut } \\
\text { grade }\end{array}$ & CD19 & $\begin{array}{l}\text { - LLA-B : taux de SG à } 1 \text { an de } 76 \% \\
\text { - Lymphome B de haut grade : durée médiane de SG } \\
\text { de } 8,3 \text { mois }\end{array}$ \\
\hline Axicabtagène ciloleucel $\dagger^{6}$ & Lymphome B de haut grade & CD19 & Taux de SG à 18 mois de $58 \%$ \\
\hline 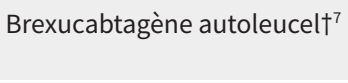 & $\begin{array}{l}\text { Lymphome à cellules du } \\
\text { manteau }\end{array}$ & CD19 & Taux de SG à 1 an de $83 \%$ \\
\hline Idécabtagène vicleucel†8 & Myélome multiple & BCMA & Durée médiane de SSP de 11,8 mois \\
\hline Lisocabtagène maraleucelł ${ }^{9}$ & Lymphome B de haut grade & CD19 & Durée médiane de SG de 18,8 mois \\
\hline
\end{tabular}

\section{De quelles données dispose-t-on jusqu'à maintenant?}

Le tableau 1 synthétise les données relatives à l'efficacité des produits de la thérapie par cellules CAR-T. Par le passé, les patients ayant une LLA-B récidivante ou réfractaire présentaient un taux de survie à 1 an d'environ $30 \%$. Les patients qui ont un lymphome $B$ de haut grade récidivant ou réfractaire et qui subissent une chimiothérapie classique ont quant à eux une durée médiane de survie globale de 6,2 mois $^{5}$. 


\section{Quels sont les préjudices associés à la thérapie par cellules CAR-T?}

Dans le mois qui suit l'administration du traitement, un suivi clinique étroit visant à déceler 2 types de toxicité est nécessaire. Le premier type de toxicité, appelé syndrome de relargage de cytokines (SRC), découle de l'activation du système immunitaire et de l'expression de cytokines inflammatoires ${ }^{10}$. Il se manifeste typiquement par de la fièvre, qui peut s'accompagner d'hypotension et d'hypoxie en cas de toxicité importante. Une atteinte avancée peut également entraîner une coagulation intravasculaire disséminée, une défaillance multiviscérale, voire le décès ${ }^{10}$. Les corticostéroïdes et le tocilizumab, un antagoniste du récepteur de l'interleukine-6 (IL-6), atténuent les symptômes du SRC. Chez les cas critiques, une hospitalisation à l'unité des soins intensifs est parfois nécessaire ${ }^{10}$. Le deuxième type de toxicité est appelé syndrome de neurotoxicité associé aux cellules immunitaires effectrices (ICANS). Ses principaux symptômes sont une difficulté à trouver ses mots, de l'aphasie et de la confusion. Une atteinte grave se traduit par une diminution de l'état de conscience, des troubles moteurs et un œdème cérébral ${ }^{10}$. La physiopathologie de l'ICANS est moins bien connue que celle du SRC, mais il est possible que l'ICANS découle de la perturbation de la barrière hématoencéphalique en contexte d'inflammation systémique grave. Bien que des lymphocytes T porteurs de récepteurs antigéniques chimériques aient été trouvés dans le liquide céphalorachidien à la suite d'une thérapie par cellules CAR-T, on ignore si ces cellules migrantes sont la cause directe de la neurotoxicité ${ }^{9}$. L'administration de corticostéroïdes constitue le principal traitement de l'ICANS. Notons que le SRC est un syndrome complexe potentiellement mortel qui nécessite une prise en charge par divers spécialistes, mais qu'il est généralement spontanément résolutif. En ce qui concerne l'ICANS, des séquelles neurologiques à long terme ont été signalées, mais la plupart des personnes atteintes se sont complètement rétablies.

La présentation du SRC ressemble souvent à celle du sepsis, un trouble qui lui est parfois concomitant. Les patients devraient donc tous être examinés et recevoir une antibiothérapie empirique à large spectre en plus d'un traitement ciblant le SRC. L'ICANS a quant à lui un tableau clinique variable. Un examen urgent visant à éliminer un problème neurologique, comme un AVC, s'impose chez tous les patients, et une consultation avec un neurologue est recommandée. Enfin, tous les patients qui reçoivent une thérapie par cellules CAR-T devraient consulter un médecin spécialisé en traitement cellulaire dans le premier mois.

La toxicité de la thérapie par cellules CAR-T peut également prendre la forme d'une cytopénie prolongée ou d'une hypoplasie lymphocytaire $B$, qui est associée à une hypogammaglobulinémie et à un risque d'infections opportunistes. La chimiothérapie immunosuppressive peut aussi entraîner la perte de toute immunité vaccinale préalablement acquise. Les médecins de premier recours doivent donc faire preuve de vigilance clinique pour reconnaître une infection opportuniste ou évitable par la vaccination chez leurs patients. La revaccination peut commencer 6 mois après l'administration d'une thérapie par cellules CAR-T, une fois les réserves de lymphocytes $B$ reconstituées. L'administration de cette thérapie pendant la pandémie de COVID-19 pose un risque accru de cas graves d'infection par le SRAS-CoV-2. On recommande donc, conformément aux énoncés de consensus nationaux et internationaux, de vacciner les personnes qui bénéficient de ce traitement. Comme les lymphocytes T utilisés sont modifiés au moyen d'un vecteur viral non réplicatif, ils ne peuvent se transformer en agents oncogènes.

\section{Que nous réserve l'avenir?}

À l'heure actuelle, il existe plus de 500 essais cliniques enregistrés sur des thérapies par cellules CAR-T pour lesquels on recrute activement des patients atteints de tumeurs malignes et non malignes, ce qui témoigne de l'innovation rapide réalisée dans le domaine. L'adoption à grande échelle de cette thérapie est toutefois limitée par des obstacles importants, dont les coûts élevés qui y sont associés. En 2018, aux États-Unis, le traitement de lymphomes B de haut grade par le tisagenlecleucel (cellules tisa) ou l'axicabtagène ciloleucel (cellules axi) coûtait 373000 US ${ }^{11}$, sans compter les soins de soutien et le séjour à l'hôpital. Une analyse de l'Agence canadienne des médicaments et des technologies de la santé a estimé qu'en cas de lymphome, il est peu probable que les cellules tisa et axi, au prix actuel, aient un rapport coût:efficacité satisfaisant à un seuil de disposition à payer de 50000 \$ CA ou de 100000 \$ CA par année de vie ajustée en fonction de la qualité ${ }^{12,13}$. Dans un système de santé publique où les ressources sont limitées, une diminution des coûts sera nécessaire pour répondre aux indications croissantes de la thérapie par cellules CAR-T. Partout au Canada, l'accès à ce traitement demeure difficile, les provinces n'ayant pas toutes finalisé les ententes juridiques et les plans d'affaires complexes essentiels à la mobilisation et à l'augmentation des ressources requises. C'est pourquoi les produits approuvés par Santé Canada ne sont pas toujours disponibles; de nombreux Canadiens et Canadiennes doivent donc être dirigés vers d'autres centres, au pays ou à l'étranger, pour les obtenir. De nouvelles stratégies, comme la fabrication en contexte clinique, le transport in vivo de gènes aux cellules immunitaires au moyen de nanoparticules (éliminant ainsi le besoin de fabriquer des cellules) et les produits allogéniques individualisés utilisant par exemple des lymphocytes $T$ de donneurs, sont en cours d'élaboration et pourraient atténuer les obstacles économiques et relatifs à l'accès en réduisant les coûts associés à la modification personnalisée des cellules.

\section{Références}

1. Brenner DR, Weir HK, Demers AA, et al. Projected estimates of cancer in Canada in 2020. CMAJ 2020;192:E199-205.

2. Gross G, Waks T, Eshhar Z. Expression of immunoglobulin-T-cell receptor chimeric molecules as functional receptors with antibody-type specificity. Proc Natl Acad Sci U S A 1989;86:10024-8.

3. Brentjens RJ, Latouche JB, Santos E, et al. Eradication of systemic B-cell tumors by genetically targeted human T lymphocytes co-stimulated by CD 80 and interleukin-15. Nat Med 2003;9:279-86.

4. Maude SL, Laetsch TW, Buechner J, et al. Tisagenlecleucel in children and young adults with B-cell lymphoblastic leukemia. N Engl J Med 2018;378:439-48.

5. Schuster SJ, Bishop MR, Tam CS, et al. Tisagenlecleucel in adult relapsed or refractory diffuse large B-cell lymphoma. N Engl J Med 2019;380:45-56.

6. Neelapu SS, Locke FL, Bartlett NL, et al. Axicabtagene ciloleucel CAR T-cell Therapy in refractory large B-Cell lymphoma. N Engl J Med 2017;377:2531-44.

7. Wang M, Munoz J, Goy A, et al. KTE-X19 CAR T-cell therapy in relapsed or refractory mantle-cell lymphoma. N Engl J Med 2020;382:1331-42.

8. Raje N, Berdeja J, Lin Y, et al. Anti-BCMA CAR T-cell therapy bb2121 in relapsed or refractory multiple myeloma. N Engl J Med 2019;380:1726-37.

9. Abramson JS, Palomba ML, Gordon LI, et al. Lisocabtagene maraleucel for patients with relapsed or refractory large B-cell lymphomas (TRANSCEND NHL 001): a multicentre seamless design study. Lancet 2020;396:839-52. 
10. Neelapu SS. Managing the toxicities of CAR T-cell therapy. Hematol Oncol 2019;37(Suppl 1):48-52.

11. Lin JK, Muffly LS, Spinner MA, et al. Cost effectiveness of chimeric antigen receptor T-cell therapy in multiply relapsed or refractory adult large B-cell lymphoma. J Clin Oncol 2019;37:2105-19.

12. CADTH optimal use report: Tisagenlecleucel for acute lymphoblastic leukemia and diffuse large B-cell lymphoma: recommendations. Ottawa: CADTH; 2019. Accessible ici : https://www.cadth.ca/sites/default/files/pdf/car-t/op0538-recommendations -report-jan2019.pdf (consulté le 10 févr. 2021).

13. CADTH optimal use report: Axicabtagene ciloleucel for large B-cell lymphoma: recommendations. Ottawa: CADTH; 2019. Accessible ici : https://www.cadth.ca/ sites/default/files/pdf/car-t/ct0002-axi-cel-recommendations-redacted report. pdf (consulté le 10 févr. 2021).
Le JAMC vous invite à soumettre vos textes pour la rubrique "Innovations » qui met en évidence les récentes avancées diagnostiques et thérapeutiques. De nouvelles utilisations de traitements existants seront également considérées. Pour la publication, les avantages de l'innovation, sa disponibilité et ses limites doivent être mis en évidence clairement, mais brièvement. Les éléments visuels (images) sont essentiels. Veuillez soumettre de brefs articles factuels (1000 mots et 5 références maximum) en ligne au http://mc.manuscriptcentral.com/cmaj ou envoyez un courriel à diane.kelsall@cmaj.ca pour échanger des idées.
Intérêts concurrents : Matthew Seftel déclare avoir reçu des honoraires de consultation de l'Agence canadienne des médicaments et des technologies de la santé, de Novartis et de Kite/Gilead. Kevin Hay a agi à titre de consultant ponctuel au sein de comités consultatifs de Kite/Gilead, de Celgene/Bristol-Myers Squibb et de Novartis. Aucun autre intérêt concurrent n'a été déclaré.

Cet article a été révisé par des pairs.

Affiliations : Programme de leucémie et de transplantation de la moelle osseuse de Colombie-Britannique (Hay); Division d'hématologie, Département de médecine (Seftel, Hay), Université de la ColombieBritannique; Société canadienne du sang (Seftel); Laboratoire Terry Fox (Hay), Institut de recherche sur le cancer de la Colombie-Britannique, Vancouver, C.-B.; Département de médecine interne (Brown), Collège de médecine Max Rady, Université du Manitoba, Winnipeg, Man.
Collaborateurs : Tous les auteurs ont contribué à la conception du travail, ont rédigé le manuscrit et en ont révisé de façon critique le contenu intellectuel important; ils ont donné leur approbation finale pour la version destinée à être publiée et assument l'entière responsabilité de tous les aspects du travail.

Propriété intellectuelle du contenu : Il s'agit d'un article en libre accès distribué conformément aux modalités de la licence Creative Commons Attribution (CC BY-NC-ND 4.0), qui permet l'utilisation, la diffusion et la reproduction dans tout médium à la condition que la publication originale soit adéquatement citée, que l'utilisation se fasse à des fins non commerciales (c.-à-d., recherche ou éducation) et qu'aucune modification ni adaptation n'y soit apportée. Voir : https://creativecommons. org/licenses/by-nc-nd/4.0/deed.fr.

Correspondance : Kevin Hay, khay@bccrc.ca 